\title{
V-Y advancement flap for repair of neglected injuries of the Achilles tendon using 2 mini-incisions
}

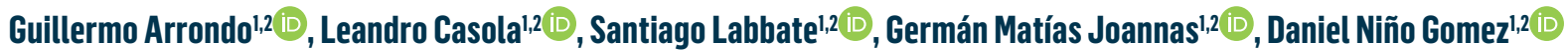 \\ 1. Instituto Dupuytren, Buenos Aires, Argentina. \\ 2. Cirujanos Especialistas en Pierna y Pie (CEPP), Buenos Aires, Argentina.
}

\begin{abstract}
Objective: To find a safe repair site for a proximal mini-incision to expose the fascia at the level of the gastrocnemius medialis (GM) myotendinous junction (MTJ).

Methods: Seventeen anatomic specimens of popliteal fossa, leg, and foot fixed in formalin were dissected, and the perpendicular distance from the apex of the medial malleolus to the GM MTJ was measured.

Results: The minimum and maximum perpendicular distances from the apex of the medial malleolus to the GM MTJ were $14.00 \mathrm{~cm}$ and $20.5 \mathrm{~cm}$, respectively. Average distance was $16.56 \mathrm{~cm}$.

Conclusion: We were able to establish a constant value and the average GM MTJ height, which allows for $V-Y$ lengthening through 2 mini-incisions. In reviewing the literature, there was no description of the approaches proposed in this study.
\end{abstract}

\section{Level of Evidence VI; Therapeutic Studies; Case Series.}

Keywords: Achilles tendon/surgery; Rupture/surgery; Tendon injuries; Fascia; Treatment outcome.

\section{Introduction}

$\mathrm{V}-\mathrm{Y}$ advancement flap was originally described for repair of neglected Achilles tendon injuries in 1975 by Abraham and Pankovich ${ }^{(1)}$ through an inverted $V$ incision which is then repaired in a $Y$ fashion to allow for end-to-end suture of the tendon.

Such technique involves an extensive posteromedial incision from the Achilles tendon distal insertion at the calcaneus which shifted medial at the proximal area to visualize the gastrocnemius medialis (GM) myotendinous junction (MTJ).

Resection of devitalized (fibrotic/scar) tissue of the neglected Achilles injury is performed, then an inverted $V$ incision of the fascia is made at the myotendinous junction, and distal traction is performed to achieve lengthening of the fascia-gastrocnemius-Achilles complex, which is subsequently reinserted into the calcaneus (chronic insertional disease) or sutured to the remaining distal portion of the healthy tendon (non-insertional chronic disease or chronic ruptures). The approach of this technique consists of a single extensive incision, which leads to complications that increase morbidity.

No study was found in the literature describing a two-incision approach: one distal incision at the site of the injury and another proximal incision near the GM MTJ.

The aim of this study was to find a safe repair site for a proximal mini-incision $(4 \mathrm{~cm})$ to expose the fascia at the level of the GM MTJ and to perform a V-Y advancement flap, thus making it possible to apply the technique using 2 mini-incisions, which reduces morbidity rates as compared to the traditional large incision.

\section{Methods}

The dissection team of the Segunda Cátedra de Anatomía at Universidad de Buenos Aires, Argentina, evaluated 17 anatomic specimens of popliteal fossa, leg, and foot fixed in $5 \%$ formalin, of which 7 were right lower limbs and 10 were left lower limbs.
Study performed at the Instituto Dupuytren, Buenos Aires, Argentina.

Correspondence: Germán Matías Joannas. 1729 Arevalo, $3^{\text {rd }}$ floor, Departament 301, Caba, Buenos Aires argentina. E-mail: germanjoannas@hotmail.com Conflicts of interest: none. Source of funding: none. Date received: August 08 , 2020. Date accepted: August 03, 2020. Online: August 30, 2020.
How to cite this article: Arrondo G, Casola L, Labbate S, Matías Joannas G, Niño Gomez D. V-Y advancement flap for repair of neglected injuries of the Achilles tendon using $\mathbf{2}$ mini-incisions. J Foot Ankle. 2020;14(2):123-5. 
The specimens were dissected, and a millimeter ruler was used to measure the perpendicular distance from the apex of the medial malleolus to the MTJ of GM (due to its lower insertion compared to gastrocnemius lateralis) with the knee at $90^{\circ}$ dorsiflexion. Measures for all specimens were then used to calculate the average distance between these two sites.

\section{Results}

Seventeen anatomic specimens of popliteal fossa, leg, and foot, of which 7 were right lower limbs and 10 were left lower limbs, were dissected and measured. There were no differences regarding sex (Table 1).

The minimum and maximum perpendicular distances from the apex of medial malleolus to the GM MTJ were $14.00 \mathrm{~cm}$ and $20.5 \mathrm{~cm}$, respectively. Average distance was $16.56 \mathrm{~cm}$. All measurements were obtained with knee of the anatomical specimen at $90^{\circ}$ dorsiflexion (Table 1).

\section{Discussion}

$\mathrm{V}-\mathrm{Y}$ advancement flap is performed to achieve an appropriate length for the Achilles tendon, thus restoring its anatomical continuity, muscle strength, and functionality, as well as patients' symmetrical and an proper one-leg raising ${ }^{(2-5)}$.

According to Us et al., $\mathrm{V}-\mathrm{Y}$ advancement flap is able reduce defects measuring from 4 to $6 \mathrm{~cm}^{(6)}$. Other studies, such as that of Kissel et al. ${ }^{(7)}$, coincide in the distance of defects (above $5 \mathrm{~cm}$ ) to be fixed using this technique. A study by Leimer et al. increased the length of fixable defects to up to $10 \mathrm{~cm}^{(8)}$.

Table 1. Measurement for each cadaveric specimen: perpendicular distance $(\mathrm{cm})$ from the apex of the medial malleolus to the gastrocnemius medialis myotendinous junction

\begin{tabular}{|cc|}
\hline Specimen & Distance (cm) \\
\hline 1 & 15.9 \\
\hline 2 & 20.0 \\
\hline 3 & 16.4 \\
\hline 4 & 16.5 \\
\hline 5 & 14.9 \\
\hline 6 & 15.2 \\
\hline 7 & 17.4 \\
\hline 8 & 19.2 \\
\hline 9 & 13.6 \\
10 & 14.4 \\
11 & 16.4 \\
\hline 12 & 16.7 \\
\hline 13 & 20.5 \\
\hline 14 & 16.2 \\
\hline 15 & 14.0 \\
\hline 16 & 16.8 \\
\hline 17 & 17.5 \\
\hline
\end{tabular}

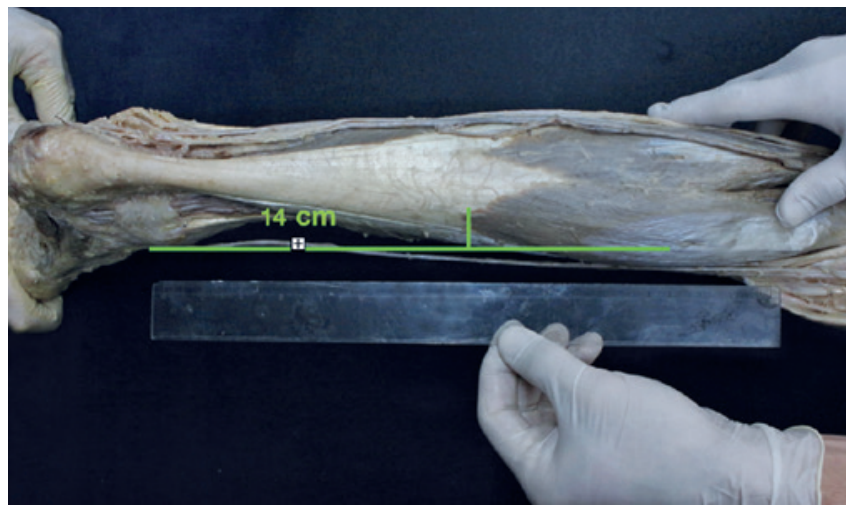

Figure 1. Image showing anatomical repair sites to be considered (internal saphenous vein and sural nerve) in the mini-incision (4-cm) approach.

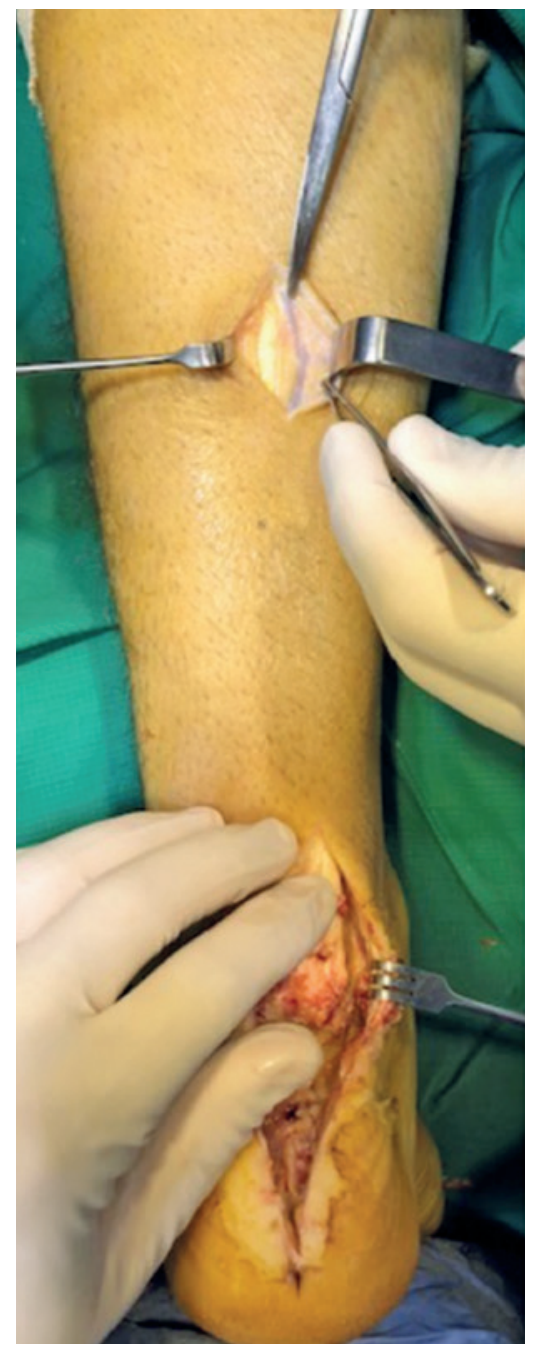

Figure 2. Mini-incision approach showing fascia with inverted $V$ incision. 
All referenced authors used a surgical approach consisting of a single extensive incision.

Based on the results of anatomical dissections and measurements, we described a safe area for proximal approach, which was established at $14 \mathrm{~cm}$ of the medial malleolus.

With the patient in the prone position, a tourniquet cuff is placed at the thigh, and enhancement is applied to the dorsal side of the foot to be operated on. A distal incision of skin and subcutaneous cell tissue is made on the Achilles tendon portion that needs repair, and the tendon is repaired.

A second approach for tendon lengthening is made at $14 \mathrm{~cm}$ from the medial malleolus and following the axis of distal incision. An incision of skin and subcutaneous cellular tissue is made, extending $2 \mathrm{~cm}$ proximally and $2 \mathrm{~cm}$ distally; furthermore, the internal saphenous vein, which runs along the sural nerve, is identified and held away (Figure 1). It is then possible to reach the fascia, where an inverted $V$ incision is made (Figure 2).

Subsequently, the subcutaneous cellular tissue of the deep fascia is detached in the portion between the two incisions to provide a greater enable distal for length to allow for distal traction of the end of the tendon that should be reinserted or sutured to the remaining tissue.

Next, the fascia is sutured with an inverted $V$ incision, which becomes a $\mathrm{Y}$ incision. Finally, the tourniquet cuff is removed, the surgical wound is closed, and the limb is protected with a walking boot walker or equine valve when applicable.

\section{Conclusion}

$\mathrm{V}-\mathrm{Y}$ advancement flap is one of the techniques for the repair of segmental defects measuring more than $5 \mathrm{~cm}$ (from 5 to $10 \mathrm{~cm}$ ) after resection of devitalized tissue in neglected injuries of the Achilles tendon.

Based on this anatomical study, we were able to establish a constant value and the average for GM MTJ height, which allows for the implementation of this technique using 2 mini-incisions, thus reducing morbidity and improving esthetic outcomes in our patients.

In reviewing the literature, there was no description of the approaches proposed in this study.

Authors' contributions: Each author contributed individually and significantly to the development of this article: GA *(https://orcid.org/0000-0001-92097660) conceived and planned the activities that led to the study, wrote the article, participated in the review process, approved the final version; LC *(https://orcid.org/0000-0003-1187-0864) wrote the article, participated in the review process; SL *(https://orcid.org/0000-0002-5212-5257) wrote the article, participated in the review process; GMJ *(https://orcid.org/O000-0001-9998-190X) wrote the article, participated in the review process; DNG *(https://orcid.org/0000-0002-7870-5882) wrote the article, participated in the review process. *ORCID (Open Researcher and Contributor ID) iD.

\section{References}

1. Abraham E, Pankovich Am. Neglected rupture of the Achiles tendon. Treatment by $\mathrm{VY}$ tendinous flap. J Bone Joint Surg Am. 1975;57(2):253-55.

2. Guclu B, Basat HC, Yildirim T, Bozduman O, Us AK. Long-term results of chronic Achilles tendon ruptures repaired with $\mathrm{V}-\mathrm{Y}$ tendon plasty and fascia turndown. Foot Ankle Int. 2016;37(7):737-42.

3. Leslie HD, Edwards WH. Neglected ruptures of the Achilles tendon. Foot Ankle Chin. 2005;10(2):357-70.

4. KL, Pavlock GS, Hecht PJ, Naselli F, Walther R. Repair of chronic Achilles tendon rupture with flexor hallux longus tendon transfer. Foot Ankle. 1993;14(8):443-9.

5. 7 Elias I, Besser M, Nazarian LN, Raikin SM. Reconstruction for missed or neglected Achilles tendon rupture with $\mathrm{V}-\mathrm{Y}$ lengthening and flexor hallucis longus tendon transfer through one incision. Foot Ankle Int. 2007;28(12):1238-48.

6. Us Ak, Bilgin SS, Aydin T, Mergen E. Repair of Neglected Achilles tendon Ruptures: procedures and functional results. Arch Orthop Trauma Surg. 1997;116 (6-7):408-11.

7. Kissel CG, Blacklidge DK, Crowley DL. Repair of neglected Achilles tendon ruptures--procedure and functional results. J Foot Ankle Surg. 1994;33(1):46-52.

8. Leimer A. Voigt C, Rachmanzdeh R. Treatment of extensive aseptic defects in old Achilles tendon ruptures: methods and case report. Foot Ankle 1992;13(4):176-80 